\title{
Fonctions zêta d'Igusa et fonctions hypergéométriques
}

\author{
par Nicusor Dan (Paris et Bucureşti)
}

Résumé. On étudie la fonction zêta d'Igusa $\zeta(P, s)$ associée à une hypersurface projective complexe $\{P=0\}$. On montre qu'elle est une intégrale d'Euler généralisée et on précise le système différentiel $A$-hypergéométrique qu'elle satisfait. On indique un algorithme pour la détermination explicite d'une équation aux différences satisfaite par $\zeta(P, s)$. On calcule explicitement cette fonction pour quelques cas particuliers. On prouve que la fonction zêta associée au résultant $R_{(1,2)}$ n'est pas une somme de produits de fonctions exponentielles et gamma.

Soit $P$ un polynôme homogène de degré $d$ en $N+1$ variables complexes. Il s'identifie à une section du fibré $O(d)$ sur $\mathbb{P}^{N}(\mathbb{C})$. On s'intéresse à la fonction de variable complexe $s$ :

$$
\zeta(P, s)=\int_{\mathbb{P}^{N}(\mathbb{C})}|P|^{2 s} d \nu_{N},
$$

où $|P|$ est la norme de $P$ pour la métrique de Fubini-Study et $d \nu_{N}$ est la mesure sur $\mathbb{P}^{N}$ invariante par l'action de $U(N+1)$, de volume 1 . Une des raisons pour cela est le fait que

$$
\frac{1}{2} \frac{d}{d s} \zeta(P, s)=\int_{\mathbb{P}^{N}(\mathbb{C})} \log |P| d \nu_{N},
$$

et la dernière quantité calcule la hauteur de l'hypersurface $\{P=0\}$ dans $\mathbb{P}^{N}$, quand $P$ est à coefficients entiers.

Suivant [GKZ], on montre au $\S 1$ que $\zeta(P, s)$ est une intégrale d'Euler généralisée. On précise le système différentiel $A$-hypergéométrique qu'elle satisfait.

1991 Mathematics Subject Classification: 11M99, 33C65, 33C70, 39A10.

Key words and phrases: Igusa zeta function, generalized Euler integral, A-hypergeometric system, difference equation. 
On sait $[\mathrm{Be}]$ que la fonction $\zeta(P, s)$ satisfait une équation

$$
\sum_{i=0}^{M} a_{i}(s) \zeta(P, s+i)=0
$$

avec $a_{i}(s)$ des polynômes non-nuls. On indique au $\S 2$ un algorithme pour la détermination explicite d'une relation (2). On donne des bornes sur $M$ et sur les degrés en $s$ des polynômes $a_{i}(s)$ en fonction de $N, d$. Cet algorithme, combiné avec celui de $[\mathrm{Pe}]$, permet de décider, étant donné un polynôme $P$, si la fonction $\zeta(P, s)$ est une somme de produits de fonctions exponentielles et gamma. Mais cette possibilité reste théorique, car l'algorithme est très long.

Une généralisation mineure de l'intégrale (1) est l'intégrale

$$
\zeta(P, s)=\int_{\mathbb{P}^{N_{1}}(\mathbb{C}) \times \ldots \times \mathbb{P}^{N_{k}}(\mathbb{C})}|P|^{2 s} d \nu_{N_{1}} \ldots d \nu_{N_{k}},
$$

où $P$ est un polynôme multi-homogène sur $\mathbb{P}^{N_{1}}(\mathbb{C}) \times \ldots \times \mathbb{P}^{N_{k}}(\mathbb{C})$ et $|P|$ est sa norme pour la métrique produit de métriques de Fubini-Study.

Le cas particulier qui a motivé ce travail est le cas où $P=R_{\left(d_{0,}, \ldots, d_{N}\right)}$, le résultant des $N+1$ polynômes en $N+1$ variables homogènes de degrés $d_{0}, \ldots, d_{N}$. L'intégrale $\zeta^{\prime}(P, 0)$ a été calculée dans [BGS] et c'est un nombre rationnel. Cela pourrait suggérer que l'intégrale $\zeta(P, s)$ est une combinaison linéaire de produits de fonctions gamma. On montre au $\S 3$ que ce n'est pas vrai pour $P=R_{(1,2)}$. Le $\S 3$ contient également des expressions de $\zeta(P, s)$ comme somme d'une série hypergéométrique généralisée dans les cas: $P=$ $R_{(1, \ldots, 1)}, P=R_{(1, n)}$ et $P$ un polynôme quadratique quelconque.

1. $\zeta(P, s)$ comme intégrale d'Euler généralisée. On fixe les coordonnées $X_{0}, \ldots, X_{N}$ sur $\mathbb{P}^{N}(\mathbb{C})$. On note $x_{i}=X_{i} / X_{0}$ pour tout $i=$ $1, \ldots, N$. Soit $P$ un polynôme homogène de degré $d$ dans les variables $X_{0}, \ldots, X_{N}$. Il s'identifie à une section du fibré $O(d)$ sur $\mathbb{P}^{N}(\mathbb{C})$. On note $|P|$ sa norme pour la métrique de Fubini-Study sur ce fibré. On note $d \nu_{N}$ la mesure sur $\mathbb{P}^{N}(\mathbb{C})$, invariante par l'action de $U(n+1)$, normalisée pour que le volume de $\mathbb{P}^{N}(\mathbb{C})$ soit 1 . L'intégrale

$$
\zeta(P, s)=\int_{\mathbb{P}^{N}(\mathbb{C})}|P|^{2 s} d \nu_{N}
$$

est bien définie pour tout nombre complexe $s$ satisfaisant $\operatorname{Re}(s)>0$, car la fonction $|P|^{2 s}$ est continue sur $\mathbb{P}^{N}(\mathbb{C})$. Elle coïncide avec l'intégrale indéfinie convergente

$$
\int_{\mathbb{P}^{N}(\mathbb{C})-\left\{X_{0}=0\right\}}|P|^{2 s} d \nu_{N}
$$


On exprime cette intégrale en coordonnées $x_{1}, \ldots, x_{N}$ :

$$
|P|^{2}=\frac{P_{0}\left(x_{1}, \ldots, x_{N}\right) \bar{P}_{0}\left(x_{1}, \ldots, x_{N}\right)}{\left(1+\left|x_{1}\right|^{2}+\ldots+\left|x_{N}\right|^{2}\right)^{d}},
$$

où $P_{0}$ est le des-homogénéisé du polynôme $P$, et ([GH], pp. 30-31)

$$
\begin{aligned}
d \nu_{N} & =N ! \omega^{N}, \\
\omega & =\frac{i}{2 \pi}\left[\frac{\sum_{j=1}^{N} d x_{j} \overline{d x_{j}}}{1+\sum_{j=1}^{N}\left|x_{j}\right|^{2}}-\frac{\left(\sum_{j=1}^{N} \bar{x}_{j} d x_{j}\right) \wedge\left(\sum_{j=1}^{N} x_{j} \overline{d x_{j}}\right)}{\left(1+\sum_{j=1}^{N}\left|x_{j}\right|^{2}\right)^{2}}\right] .
\end{aligned}
$$

Au total,

$$
\zeta(P, s)=N !\left(\frac{i}{2 \pi}\right)^{N} \int_{\mathbb{C}^{N}}\left(P_{0} \bar{P}_{0}\right)^{s} \frac{d x_{1} \overline{d x_{1}} \ldots d x_{N} \overline{d x_{N}}}{\left(1+\left|x_{1}\right|^{2}+\ldots+\left|x_{N}\right|^{2}\right)^{d s+N+1}} .
$$

Pour tout $j=1, \ldots, N$, on écrit $x_{j}=t_{2 j-1}+i t_{2 j}$ pour $t_{1}, \ldots, t_{2 N}$ des variables réelles. On note $P_{1}$ le polynôme de degré $2 d$ dans les variables $t_{1}, \ldots, t_{2 N}$ obtenu à partir du polynôme $P_{0} \bar{P}_{0}$. Il en résulte que

$$
\begin{aligned}
& \zeta(P, s) \\
& =N ! \frac{1}{\pi^{N}} \int_{\mathbb{R}^{2 N}} P_{1}\left(t_{1}, \ldots, t_{2 N}\right)^{s}\left(1+t_{1}^{2}+\ldots+t_{2 N}^{2}\right)^{-d s-N-1} d t_{1} \ldots d t_{2 N} .
\end{aligned}
$$

On rappelle la définition suivante de [GKZ]:

Soient $P_{1}, \ldots, P_{m}$ des polynômes de Laurent dans $\mathbb{C}^{k}$ et $\alpha_{1}, \ldots, \alpha_{m}$, $\beta_{1}, \ldots, \beta_{k}$ des nombres complexes. Soit $U$ l'ouvert de $\mathbb{C}^{k}$ défini par $x_{i} \neq 0$ pour tout $i=1, \ldots, k$ et $P_{j} \neq 0$ pour tout $j=1, \ldots, m$. Soit $L$ le système local sur $U$ ayant les exposants de monodromie $\alpha_{j}$ autour de $\left\{P_{j}=0\right\}$ et $\beta_{i}$ autour de $\left\{x_{i}=0\right\}$ et soit $\sigma$ un $k$-cycle singulier avec les coefficients dans L. Alors

$$
\int_{\sigma} \prod_{j} P_{j}\left(x_{1}, \ldots, x_{k}\right)^{\alpha_{j}} x_{1}^{\beta_{1}} \ldots x_{k}^{\beta_{k}} d x_{1} \ldots d x_{k}
$$

s'appelle une intégrale d'Euler généralisée.

Le $k$-cycle singulier peut être fini ou seulement localement fini. Dans le dernier cas, l'intégrale doit être convergente.

L'intégrale (3) est une intégrale d'Euler généralisée. En effet, on prend $k=2 N$, on considère l'inclusion canonique $\mathbb{R}^{2 N} \subset \mathbb{C}^{2 N}$ et on prolonge $t_{1}, \ldots, t_{2 N}$ en coordonnées complexes sur $\mathbb{C}^{2 N}$.

On considère dans la définition $m=2, P_{1}=P_{1}\left(t_{1}, \ldots, t_{2 N}\right), P_{2}=$ $1+t_{1}^{2}+\ldots+t_{2 N}^{2}, \alpha_{1}=s, \alpha_{2}=-d s-N-1, \beta_{1}=\ldots=\beta_{2 N}=0$. On prend l'ouvert $U$ et le système local $L$ comme dans la définition. Le système local $L$ restreint à $\mathbb{R}^{2 N}$ est trivial, car les polynômes $P_{1}, P_{2}$ sont à coefficients réels. 
On considère une triangulation localement finie de $\mathbb{R}^{2 N} \cap U=\mathbb{R}^{2 N}$ $\left\{P_{1}=0\right\}-\bigcup_{i=1}^{2 N}\left\{t_{i}=0\right\}$ et on note $\sigma$ le $2 N$-cycle qui lui est associé. Comme son support est inclus dans $\mathbb{R}^{2 N}$, il est bien un $2 N$-cycle pour le système local $L$. Avec toutes ces notations, l'intégrale (3) s'écrit

$$
\frac{N !}{\pi^{N}} \int_{\sigma} P_{1}^{\alpha_{1}} P_{2}^{\alpha_{2}} t_{1}^{\beta_{1}} \ldots t_{2 N}^{\beta_{2 N}} d t_{1} \ldots d t_{2 N} .
$$

Suivant [GKZ], on va décrire un système différentiel satisfait par une intégrale générale de type (3). Pour cela, on note

$$
P_{1}=\sum_{I \in \mathcal{I}} B_{I} t^{I}, \quad P_{2}=A_{0}+\sum_{i=1}^{2 N} A_{i} t_{i}^{2},
$$

où $\mathcal{I}$ est l'ensemble des $2 N$-uples $I=\left(I_{1}, \ldots, I_{2 N}\right)$ vérifiant $I_{1} \geq 0, \ldots$, $I_{2 N} \geq 0, \sum_{i=1}^{2 N} I_{i} \leq 2 \alpha$. On note $\underline{0}$ le $2 N$-uple $(0, \ldots, 0)$. On suppose $A_{i}>0$ pour tout $i=0, \ldots, 2 N$.

Proposition 1.1. Soient $P_{1}, P_{2}$ les polynômes définis par (5) et $s$ un nombre complexe vérifiant $\operatorname{Re}(s)>2$. Alors l'intégrale

$$
I\left(A_{i}, B_{I}\right)=\int_{\mathbb{R}^{2 N}} P_{1}^{s} P_{2}^{-d s-N-1} d t_{1} \ldots d t_{2 N}
$$

vérifie les équations différentielles suivantes, dans les variables $A_{i}, B_{I}$ :

$$
\begin{gathered}
\left(\sum_{I \in \mathcal{I}} B_{I} \frac{\partial}{\partial B_{I}}\right) I\left(A_{i}, B_{I}\right)=s I\left(A_{i}, B_{I}\right), \\
\left(\sum_{j=0}^{2 N} A_{j} \frac{\partial}{\partial A_{j}}\right) I\left(A_{i}, B_{I}\right)=(-d s-N) I\left(A_{i}, B_{I}\right), \\
\left(\sum_{I \in \mathcal{I}} I_{i} B_{I} \frac{\partial}{\partial B_{I}}+2 A_{i} \frac{\partial}{\partial A_{i}}\right) I\left(A_{i}, B_{I}\right)=-I\left(A_{i}, B_{I}\right)
\end{gathered}
$$

pour tout $i=1, \ldots, 2 N$, et

$$
\left(\frac{\partial^{I_{1}+\ldots+I_{2 N}}}{\partial A_{1}^{I_{1}} \ldots \partial A_{2 N}^{I_{2 N}}} \frac{\partial^{2}}{\partial B_{0}^{2}}\right) I\left(A_{i}, B_{I}\right)=\left(\frac{\partial^{I_{1}+\ldots+I_{2 N}}}{\partial A_{0}^{I_{1}+\ldots+I_{2 N}}} \frac{\partial^{2}}{\partial B_{I}^{2}}\right) I\left(A_{i}, B_{I}\right)
$$

pour tout $I \in \mathcal{I}, I \neq \underline{0}$.

Preuve. La preuve coïncide avec la preuve du théorème (2.7) dans [GKZ]; il faut seulement prouver que les intégrales qui apparaissent dans les diverses identités sont convergentes.

On note $D(R)$ la boule fermée, dans $\mathbb{R}^{2 N}$, centrée à l'origine, de rayon $R$.

Le polynôme $P_{2}$ est partout positif, car $A_{i}>0$ pour $i=0, \ldots, 2 N$. Il résulte que la fonction $P_{2}^{-d s-N-1}$ est de classe $C^{\infty}$ sur $\mathbb{R}^{2 N}$. Comme $\operatorname{Re}(s)>2$, la fonction $P_{1}^{s}$ est de classe $C^{2}$ sur $\mathbb{R}^{2 N}$. Il résulte que les dérivées 
d'ordre au plus 2 dans les variables $B_{I}$ et de tout ordre dans les variables $A_{i}$ commutent avec l'intégrale sur $D(R)$ pour la forme $P_{i}^{s} P_{2}^{-d s-N-1} d t_{1} \ldots d t_{2 N}$.

On a

$$
\begin{aligned}
\left(\sum_{I} B_{I} \frac{\partial}{\partial B_{I}}\right) & \int_{D(R)} P_{1}^{s} P_{2}^{-d s-N-1} d t_{1} \ldots d t_{2 N} \\
& =\int_{D(R)} s P_{1}^{s-1}\left(\sum_{I \in J} B_{I} \frac{\partial P_{1}}{\partial B_{I}}\right) P_{2}^{-d s-N-1} d t_{1} \ldots d t_{2 N} \\
& =\int_{D(R)} P_{1}^{s-1} P_{1} P_{2}^{-d s-N-1} d t_{1} \ldots d t_{2 N} .
\end{aligned}
$$

De même,

$$
\begin{aligned}
\sum_{j=0}^{2 N}\left(A_{j} \frac{\partial}{\partial A_{j}}\right) & \int_{D(R)} P_{1}^{s} P_{2}^{-d s-N-1} d t_{1} \ldots d t_{2 N} \\
& =(-d s-N-1) \int_{D(R)} P_{1}^{s} P_{2}^{-d s-N-1} d t_{1} \ldots d t_{2 N}
\end{aligned}
$$

$$
\begin{aligned}
& \left(\frac{\partial^{I_{1}+\ldots+I_{2 N}}}{\partial A_{1}^{I_{1}} \ldots A_{2 N}^{I_{2 N}}} \frac{\partial^{2}}{\partial B_{0}^{2}}\right) \int_{D(R)} P_{1}^{s} P_{2}^{-d s-N-1} d t_{1} \ldots d t_{2 N} \\
= & \left(\frac{\partial^{I_{1}+\ldots+I_{2 N}}}{\partial A_{0}^{I_{1}+\ldots+I_{2 N}}} \frac{\partial^{2}}{\partial B_{I}^{2}}\right) \int_{D(R)} P_{1}^{s} P_{2}^{-d s-N-1} d t_{1} \ldots d t_{2 N}
\end{aligned}
$$$$
=\int_{D(R)} s(s-1) P_{1}^{s-2}
$$

$$
\times \prod_{i=1}^{I_{1}+\ldots+I_{N}}(-d s-N-i) \cdot P_{2}^{-d s-N-I_{1}-\ldots-I_{2 N}} t_{1}^{2 I_{1}} \ldots t_{2 N}^{2 I_{2 N}} d t_{1} \ldots d t_{2 N},
$$

$$
\begin{aligned}
& \left(\sum_{I \in \mathcal{I}} I_{i} B_{I} \frac{\partial}{\partial B_{I}}+2 A_{i} \frac{\partial}{\partial A_{i}}+1\right) \int_{D(R)} P_{1}^{s} P_{2}^{-d s-N-1} d t_{1} \ldots d t_{2 N} \\
& =\int_{D(R)}\left[s P_{1}^{s-1}\left(\sum_{I \in \mathcal{I}} I_{i} B_{I} t^{I}\right) P_{2}^{-d s-N-1}\right] d t_{1} \ldots d t_{2 N} \\
& \quad+\int_{D(R)}\left[(-d s-N-1) P_{2}^{-d s-N-2} 2 t_{i}^{2}+P_{1}^{s} P_{2}^{-d s-N-1}\right] d t_{1} \ldots d t_{2 N} \\
& =\int_{D(R)} d\left[t_{i} P_{1}^{s} P_{2}^{-d s-N-1} d t_{1} \ldots \widehat{d t_{i}} \ldots d t_{2 N}\right]
\end{aligned}
$$




$$
=\int_{\partial D(R)} t_{i} P_{1}^{s} P_{2}^{-d s-N-1} d t_{1} \ldots \widehat{d t}_{i} \ldots d t_{2 N} .
$$

Le polynôme $P_{2}$ est minoré par un polynôme $M\left(1+t_{1}^{2}+\ldots+t_{2 N}^{2}\right)$, pour une constante positive $M$. Il en résulte que $P_{1} P_{2}^{-d}$ est borné sur $\mathbb{R}^{2 N}$.

Le fait que

$$
\frac{d t_{1} \ldots d t_{2 N}}{\left(1+t_{1}^{2}+\ldots+t_{2 N}^{2}\right)^{N+1 / 2}} d t_{1} \ldots \widehat{d t}_{i} \ldots d t_{2 N}
$$

est intégrable sur $\mathbb{R}^{2 N}$ implique que toutes les intégrales sur $D(R)$ dans les expressions (10)-(13) convergent vers les intégrales similaires sur $\mathbb{R}^{2 N}$ quand $R \rightarrow \infty$.

La dernière intégrale de (13) est bornée par

$$
\int_{\partial D(R)} \frac{\sqrt{1+t_{1}^{2}+\ldots+t_{2 N}^{2}}}{\left(1+t_{1}^{2}+\ldots+t_{2 N}^{2}\right)^{N+1}} d t_{1} \ldots \widehat{d t}_{i} \ldots d t_{2 N}
$$

qui converge vers 0 quand $R \rightarrow \infty$.

Donc (6)-(9) résultent des relations (10)-(13) pour $R \rightarrow \infty$.

On va préciser dans la suite une base de solutions pour le système d'équations (6)-(9) sur un ouvert de l'espace des paramètres $A_{i}, B_{I}$, en explicitant la construction de [GZK] dans notre exemple. On commence par donner les définitions et les faits suivants de [GZK].

Soit $A$ un ensemble de vecteurs $\chi_{k}=\left(\chi_{i k}\right)_{1 \leq i \leq n}, k \in M$, dans un hyperplan affin primitif de $\mathbb{Z}^{n}$ (i.e. la préimage de 1 par une application linéaire $\mathbb{Z}^{n} \rightarrow \mathbb{Z}$ ), qui engendrent $\mathbb{Z}^{n}$.

Soit $L$ le réseau des relations entre les vecteurs $\chi_{k}: a=\left(a_{k}\right)_{k \in M} \in L$ si $\sum_{k \in M} a_{k} \chi_{i k}=0$ pour tout $i=1, \ldots, n$.

Soient $\beta_{1}, \ldots, \beta_{n}$ des nombres complexes. On note $v_{k}, k \in M$, les coordonnées sur $\mathbb{C}^{M}$. Le système hypergéométrique associé à l'ensemble $A$ et à l'ensemble des exposants $\beta_{i}$ est le système d'équations différentielles suivant sur $\mathbb{C}^{M}$ :

$$
\left(\sum_{k \in M} \chi_{i k} v_{k} \frac{\partial}{\partial v_{k}}\right) I(v)=\beta_{i} I(v)
$$

pour $i=1, \ldots, n$,

$$
\prod_{a_{k}>0}\left(\frac{\partial}{\partial v_{k}}\right)^{a_{k}} I(v)=\prod_{a_{k}<0}\left(\frac{\partial}{\partial v_{k}}\right)^{-a_{k}} I(v)
$$

pour tout $a=\left(a_{k}\right) \in L$.

Soit $\gamma=\left(\gamma_{k}\right), k \in M$, un vecteur dans $\mathbb{C}^{M}$. La série formelle

$$
\Phi_{\gamma}(v)=\sum_{a \in L} \prod_{k \in M} v_{k}^{a_{k}+\gamma_{k}} / \prod_{k \in M} \Gamma\left(\gamma_{k}+a_{k}+1\right)
$$


est une solution formelle pour le système d'équations (14), (15) si $\gamma$ vérifie

$$
\sum_{k \in M} \chi_{i k} \gamma_{k}=\beta_{i}
$$

pour tout $i=1, \ldots, n$.

Un sous-ensemble $K \subset M$ s'appelle base si l'ensemble des vecteurs $\chi_{k}, k \in K$, forment une base dans $\mathbb{R}^{n}=\mathbb{Z}^{n} \otimes_{\mathbb{Z}} \mathbb{R}$. Pour une base $K$ on note $\Pi_{\mathbb{Z}}(\beta, K)$ l'ensemble des vecteurs $\gamma \in \mathbb{C}^{M}$ vérifiant (17) et la condition $\gamma_{k} \in \mathbb{Z}$ si $k \notin K$.

On introduit l'espace "logarithmique" $\mathbb{R}^{M}$ avec les coordonnées $w_{k}, k \in$ $M$. Pour toute base $K \subset M$ et tout vecteur $w \in \mathbb{R}^{M}$ on définit la fonction linéaire $\varphi_{K, w}$ sur $\mathbb{R}^{n}=\mathbb{Z}^{n} \otimes_{\mathbb{Z}} \mathbb{R}$ par $\varphi_{K, w}\left(\chi_{k}\right)=w_{k}$ si $k \in K$. On note $C(K)$ le cône dans $\mathbb{R}^{M}$ défini par les inégalités $\varphi_{K, w}\left(\chi_{k}\right)<w_{k}$ pour $k \notin K$.

La proposition 2 de [GKZ] affirme que pour toute base $K$ et pour tout vecteur $\gamma \in \Pi_{\mathbb{Z}}(\beta, K)$ il existe un vecteur $C \in C(K)$ tel que la série (16) converge pour $\left(-\ln \left|v_{k}\right|\right)_{k \in M} \subset C+C(K)$.

Les séries $\Phi_{\gamma}(v)$ et $\Phi_{\gamma^{\prime}}(v)$ sont les mêmes si $\gamma-\gamma^{\prime} \in L$. L'ensemble de ces séries est donc paramétrisé par l'ensemble $\Pi_{\mathbb{Z}}(\beta, K) / L$.

Soit $\Delta(K)$ la clôture convexe dans $\mathbb{R}^{n}$ de l'ensemble des vecteurs $\chi_{k}$, $k \in M$, et du vecteur 0 . Le cardinal de l'ensemble $\Pi_{\mathbb{Z}}(\beta, K) / L$ est égal à $n ! \operatorname{vol}(\Delta(K))$, où $\operatorname{vol}(\Delta(K))$ est le volume canonique dans $\mathbb{R}^{n}=\mathbb{Z}^{n} \otimes \mathbb{R}$.

On note $P$ la clôture convexe dans $\mathbb{R}^{n}$ de l'ensemble des vecteurs $\chi_{j}$, $j \in M$, et du vecteur 0 . On appelle triangulation du polyèdre $P$ un ensemble $T$ de bases $K$ satisfaisant aux conditions $\bigcup_{K \in T} \Delta(K)=P$ et pour tous $K_{1}, K_{2} \in T, \Delta\left(K_{1}\right) \cap \Delta\left(K_{2}\right)$ est une face (peut-être vide) de $\Delta\left(K_{1}\right)$ et de $\Delta\left(K_{2}\right)$.

Une triangulation $T$ s'appelle régulière si le cône $C(T)=\bigcap_{K \in T} C(K)$ est non-vide. On dit que la triangulation $T$ et l'ensemble des exposants $\beta_{i}$ satisfont la condition de non-résonnance si les ensembles $\Pi_{\mathbb{Z}}(\beta, K)$ sont deux à deux disjoints pour $K \in T$.

Pour une triangulation qui satisfait la condition de non-résonnance, on note $\Pi(T)$ la réunion $\bigcup_{K \in T} \Pi_{\mathbb{Z}}(\beta, K) / L$. Le résultat principal de [GZK] est le suivant :

Soit $T$ une triangulation régulière et satisfaisant la condition de nonrésonnance par rapport aux exposants $\beta_{i}$. Alors il existe un vecteur $C \in C(T)$ tel que l'ensemble des fonctions $\Phi_{\gamma}(v)$, pour $\gamma \in \Pi(T)$, forment une base de solutions du système des équations (14), (15) sur l'ouvert de $\mathbb{C}^{M}$ défini par

$$
\left(-\ln \left|v_{k}\right|\right)_{k \in M}=C+C(T) .
$$

Dans notre exemple $n=2+2 N, M=\{0, \ldots, 2 N\} \cup \mathcal{I}$; les coordonnées $v_{k}$ sont $A_{i}$ pour $k=i \in\{0, \ldots, 2 N\}$ et $B_{I}$ pour $k=I \in \mathcal{I}$. On rappelle que $\mathcal{I}$ est l'ensemble des $2 N$-uples $I=\left(I_{1}, \ldots, I_{2 N}\right)$ vérifiant $\sum_{i=1}^{2 N} I_{i} \leq 2 d$. 
On fixe une base $e_{-2}, e_{-1}, e_{1}, \ldots, e_{2 N} \operatorname{sur} \mathbb{Z}^{2} \times \mathbb{Z}^{2 N}$. On fait la convention $e_{0}=0$. L'ensemble $A$ est formé des vecteurs $\chi_{I}=e_{-2}+I$ pour $I \in \mathcal{I}$ et des vecteurs $\chi_{i}=e_{-1}+2 e_{i}$ pour $i=0, \ldots, 2 N$. Les exposants sont $\beta_{-2}=s$, $\beta_{-1}=-d s-N, \beta_{1}=\ldots=\beta_{2 N}=-1$.

L'ensemble des équations (14) est donné par l'équation (6) pour $i=-2$, par l'équation (7) pour $i=-1$ et par l'ensemble des équations (8) pour $i=1, \ldots, 2 N$. L'ensemble des équations (9) représentent l'ensemble des équations (15) pour une base de $L$. Elles sont équivalentes à l'ensemble des équations (15) pour tous $a \in L$.

Les équations (17) s'écrivent

$$
\begin{gathered}
\sum_{i=0}^{2 N} \gamma_{i}=s, \\
\sum_{I \in \mathcal{I}} \gamma_{I}=-d s-N, \\
2 \gamma_{i}+\sum_{I \in \mathcal{I}} I_{i} \gamma_{I}=-1
\end{gathered}
$$

pour tout $i=1, \ldots, 2 N$.

Pour tout $i=0, \ldots, 2 N$, on note $\underline{i}$ l'élément $2 d e_{i} \in \mathcal{I}$. La clôture convexe $P$ de l'ensemble des vecteurs $A$ et du vecteur 0 est le polyèdre convexe de sommets 0 et $\chi_{\underline{i}}=e_{-2}+\underline{i}, \chi_{i}=e_{-1}+2 e_{i}$, pour $i=0, \ldots, 2 N$. C'est un cône qui a le sommet 0 et la base une prisme de bases $\chi_{0}, \ldots, \chi_{2 N}$ et $\chi_{\underline{0}}, \ldots, \chi_{\underline{2 N}}$. On choisit la triangulation de $T$ formée des bases $K_{i}=$ $\{0,1, \ldots, i, \underline{i}, \ldots, \underline{2 N}\}$ pour $i=0, \ldots, 2 N$. Un calcul évident donne $(2+$ $2 N) ! \operatorname{vol}\left(\Delta\left(K_{i}\right)\right)=2^{i}(2 d)^{N-i}$.

Proposition 1.2. La triangulation $T$ est régulière. Plus précisément, le cône $C(T)$ contient le cône non-vide $C^{\prime}(T)$ de $\mathbb{R}^{M}$ donné par les inégalités

$$
w_{I}>\left(1-\sum_{i=1}^{2 N} I_{i} /(2 d)\right) w_{\underline{0}}+\sum_{i=1}^{2 N} I_{i} /(2 d) \cdot w_{\underline{i}}
$$

pour tout $I \in \mathcal{I}-\{\underline{0}, \ldots, \underline{2 N}\}$ et

$$
d\left(w_{i+1}-w_{i}\right)>w_{\underline{i+1}}-w_{\underline{i}}
$$

pour tout $i=0, \ldots, 2 N-1$.

Preuve. La relation évidente $\chi_{\underline{i}}-\chi_{\underline{j}}=d\left(\chi_{i}-\chi_{j}\right)$ implique

$$
\varphi_{K_{i}, w}\left(\chi_{j}\right)=w_{i}+\frac{1}{d}\left(w_{\underline{j}}-w_{\underline{i}}\right)
$$

pour $j>i$ et

$$
\varphi_{K_{i}, w}\left(\chi_{\underline{j}}\right)=w_{\underline{i}}+d\left(w_{j}-w_{i}\right)
$$


pour $j<i$. Il en résulte que l'ensemble des inégalités $\varphi_{K_{i}, w}\left(\chi_{k}\right)<w_{k}$ pour $k \in\{0, \ldots, 2 N\} \cup\{\underline{0}, \ldots, \underline{2 N}\}-K_{i}$ coïncide avec l'ensemble des inégalités

$$
d\left(w_{j}-w_{i}\right)>w_{\underline{j}}-w_{\underline{i}}
$$

pour tous $j>i$. Elles sont évidemment équivalentes à l'ensemble des inégalités (23).

Soit $K_{i}$ une base de $T$ et $I \in \mathcal{I}-\{\underline{0}, \ldots, \underline{2 N}\}$. L'inégalité (22) appliquée à $I$ et l'ensemble des inégalités $\varphi_{K_{i}, w}\left(\chi_{j}\right) \leq w_{\underline{j}}$ pour tout $j \in\{0, \ldots, 2 N\}$ impliquent $\varphi_{K_{i}, w}\left(\chi_{I}\right)<w_{I}$, d'où la conclusion.

Proposition 1.3. Soit s un nombre complexe vérifiant $2 d s \notin \mathbb{Z}$. Alors la triangulation $T$ et les exposants $\beta_{-2}=s, \beta_{-1}=-d s-N, \beta_{1}=\ldots=$ $\beta_{2 N}=-1$ satisfont la condition de non-résonnance.

Preuve. Soient $i, j \in\{0, \ldots, 2 N\}, i<j$. On suppose par l'absurde qu'il existe un élément $\gamma \in \Pi_{\mathbb{Z}}\left(\beta, K_{i}\right) \cap \Pi_{\mathbb{Z}}\left(\beta, K_{j}\right) \subset \mathbb{C}^{M}$. Il en résulte que $\gamma_{k} \in \mathbb{Z}$ pour tout $k \in M-(\{0, \ldots, i\} \cup\{\underline{j}, \ldots, \underline{2 N}\})$. Les équations (21) appliquées pour $l=j, \ldots, 2 N$ donnent $2 \overline{d \gamma_{\underline{l}}} \in \mathbb{Z}$. L'équation (19) donne $\sum_{l=j}^{2 N} \gamma_{\underline{l}}-s \in \mathbb{Z}$, d'où $2 d s \in \mathbb{Z}$, contradiction.

La relation (18) pour $C^{\prime}(T)$ équivaut aux inégalités

$$
\begin{gathered}
\left|B_{I}\right|^{2 d}<C_{I}\left|B_{\underline{0}}\right|^{2 d-\sum_{i=1}^{2 N} I_{i}} \prod_{i=0}^{2 N}\left|B_{\underline{i}}\right|^{I_{i}}, \\
\left|B_{\underline{i+1}} / B_{\underline{i}}\right|>C_{i}\left|A_{i+1} / A_{i}\right|^{d}
\end{gathered}
$$

pour certaines constantes positives $C_{I}$ pour $I \in \mathcal{I}-\{\underline{0}, \ldots, \underline{2 N}\}$ et $C_{i}$ pour $i=0, \ldots, 2 N-1$.

Le résultat principal de [GZK] et les propositions 1.2 et 1.3 impliquent :

Proposition 1.4. L'ensemble des fonctions $\Phi_{\gamma}\left(A_{i}, B_{I}\right)$ pour $\gamma \in \Pi(T)$ forment une base de solutions du système d'équations (6)-(9) sur l'ouvert défini par les inégalités (27) de l'espace des paramètres $A_{i}, B_{I}$.

Corollaire 1.5. Il existe un ensemble de fonctions complexes $f_{\gamma}(s)$, pour $\gamma \in \Pi(T)$, holomorphes sur $\mathbb{C}-\frac{1}{2 d} \mathbb{Z}$, telles que

$$
I\left(A_{i}, B_{I}\right)=\sum_{\gamma \in \Pi(T)} f_{\gamma}(s) \Phi_{\gamma}\left(A_{i}, B_{I}\right)
$$

sur l'ouvert de l'espace des paramètres $A_{i}, B_{I}$ défini par les inégalités (27).

La détermination explicite des fonctions $f_{\gamma}(s)$ paraît une question très difficile. 
3. Une relation de récurrence pour $\zeta(P, s)$

THÉORÈme 2.1. La fonction $\zeta(P, s)$ satisfait une relation

$$
\sum_{i=0}^{M} b_{i}(s) \zeta(P, s+i)=0
$$

où :

(a) $M$ est un entier vérifiant

$$
M \leq\left(\begin{array}{c}
d+N(d-1) \\
N
\end{array}\right)^{2},
$$

(b) $b_{i}(s)$ sont des fonctions rationnelles, pas toutes nulles, de la forme

$$
b_{i}(s)=\prod_{k=1}^{i}(s-E+k)^{-2 k} \cdot \prod_{k=i+1}^{M}(s-E+k)^{-2 k+2} \cdot a_{i}(s),
$$

où $E$ est l'entier défini par la relation (44) ci-dessous et $a_{i}$ sont des polynômes de degré au plus $M(M-1)+2 i$ en $s$ dont les coefficients sont des fonctions polynômiales des coefficients du polynôme $P$ et de leurs conjugués.

REMARQues. 1) La démonstration fournit un algorithme pour le calcul de l'entier $M$ et des fonctions $a_{i}(s)$. Il est malheureusement très longue dans presque tous les cas.

2) Un énoncé analogue est valable pour l'intégrale d'une puissance d'un polynôme homogène sur la sphère $S^{n} \subset \mathbb{R}^{n+1}$.

3) Ce théorème a été prouvé pour $d=1$ dans $[\mathrm{Ca}-\mathrm{M}]$ et pour $d=2$ dans $[\mathrm{Be}-\mathrm{Y}]$.

Pre u ve (du théorème 2.1). On considère la famille de polynômes

$$
P_{t}=(1-t) P+t\left(X_{0}^{d}+\ldots+X_{N}^{d}\right)
$$

pour $t \in \mathbb{R}$. Il suffit de prouver théorème pour $P_{t}$ avec $t$ générique au sens de Zariski. En effet, le théorème implique que la fonction

$$
K_{t}(s)=\int_{\mathbb{P}^{N}(\mathbb{C})}\left|P_{t}\right|^{2 s} d \nu_{N}
$$

vérifie

$$
\sum_{i=0}^{M} b_{i, t}(s) K_{t}(s+i)=0
$$

où

$$
b_{i, t}(s)=\prod_{k=1}^{i}(s-E+k)^{-2 k} \cdot \prod_{k=i+1}^{M}(s-E+k)^{-2 k+2} \cdot a_{i, t}(s)
$$


et $a_{i, t}(s)$ sont des fonctions polynômiales en $s, t=\bar{t}$, en les coefficients du polynôme $P$ et en leurs conjugués.

On note $r_{i}=a_{i, t}(t), r$ le minimum des $r_{i}$ pour $i=0, \ldots, M$, et on définit

$$
a_{i}(s)=\lim _{t>0, t \rightarrow 0} a_{i, t}(s) / t^{r} .
$$

Les fonctions $a_{i}(s)$ sont des polynômes en $s$ et en les coefficients du polynôme $P$ et leurs conjugués. Elles ne sont pas toutes nulles.

La relation (23) et la relation évidente

$$
\lim _{t>0, t \rightarrow 0} \int_{\mathbb{P}^{N}(\mathbb{C})}\left|P_{t}\right|^{2 s} d \nu_{N}=\int_{\mathbb{P}^{N}(\mathbb{C})}|P|^{2 s} d \nu_{N}=\zeta(P, s)
$$

impliquent (28). Le degré en $s$ de $a_{i}(s)$ n'est pas plus grand que le degré en $s$ de $a_{i, t}(s)$ pour $t$ générique.

Le théorème découle des deux propositions suivantes :

Proposition 2.2. Il existe :

(a) un entier positif $M$ vérifiant

$$
M \leq\left(\begin{array}{c}
d+N(d-1) \\
N
\end{array}\right)^{2}
$$

(b) des fonctions méromorphes $I_{1}(s)=K_{t}(s), I_{2}(s), \ldots, I_{M}(s)$,

(c) une matrice $A(s)=\left(a_{i j}(s)\right)_{1 \leq i, j \leq M}$, avec $a_{i j}(s)$ des polynômes en $s$ de degré au plus 2 , dont les coefficients sont des fonctions polynômiales des coefficients de $P_{t}$ et de leurs conjugués,

telles que

$$
\left(\begin{array}{c}
I_{1}(s) \\
\vdots \\
I_{M}(s)
\end{array}\right)=(s-E+1)^{-2} A(s)\left(\begin{array}{c}
I_{1}(s+1) \\
\vdots \\
I_{M}(s+1)
\end{array}\right)
$$

où l'entier E est défini par la formule (44) ci-dessous.

Proposition 2.3. Soient $I_{1}(s), \ldots, I_{M}(s)$ des fonctions méromorphes et $A(s)=\left(a_{i j}(s)\right)_{1 \leq i, j \leq M}$ une matrice à coefficients polynômes de degré au plus 2 telles que (34) est vérifiée. Alors $I_{1}(s)$ vérifie

$$
\sum_{i=0}^{M} b_{i}(s) I_{1}(s+i)=0,
$$

où les $b_{i}$ sont des fonctions rationnelles données par $(30)$, avec $a_{i}(s)$ des expressions polynômiales de fonctions $a_{k j}(s), \ldots, a_{k j}(s+M)$, pas toutes nulles, et de degré en s au plus $M(M-1)+2 i$. 
Preuve de la proposition 2.2

Lemme 2.4. Pour un paramètre générique $t \in \mathbb{R}$, l'idéal engendré par les dérivées partielles $\left(P_{t}\right)_{X_{i}}$ contient tous les monômes de degré au moins $(N+1)(d-2)+1$ dans l'anneau $\mathbb{C}\left[X_{0}, \ldots, X_{N}\right]$.

Preuve. La condition que tous les monômes d'un degré fixé appartiennent à l'idéal engendré par les dérivées partielles $\left(P_{t}\right)_{X_{i}}$ s'écrit comme la non-nullité d'un déterminant dans un ensemble de déterminants dépendant des coefficients du polynôme $P_{t}$. Il suffit de prouver que ce déterminant est non-nul pour $t=1$ pour qu'il soit non-nul pour $t$ générique.

D'autre part, $P_{1}=X_{0}^{d}+\ldots+X_{N}^{d}$ et $\left(P_{1}\right)_{X_{i}}=d X_{i}^{d-1}$ pour tout $i=$ $0, \ldots, M$. Il est clair que tout monôme de degré au plus $(N+1)(d-2)+1$ contient $X_{i}^{d-1}$ pour un certain $i \in\{0, \ldots, M\}$, donc il appartient à l'idéal engendré par les $\left(P_{1}\right)_{X_{i}}$.

Par $[\mathrm{Ca}-\mathrm{M}]$,

$$
\int_{\mathbb{P}^{N}}\left|P_{t}\right|^{2 s} d \nu_{N}=\frac{\Gamma(N+1)}{\Gamma(N+1+s d)} \int_{\mathbb{C}^{N+1}}\left|P_{t}\right|^{2 s} e^{-S} d \mu
$$

où

$$
\begin{aligned}
S & =\left|X_{0}\right|^{2}+\ldots+\left|X_{N}\right|^{2}, \\
d \mu & =\left(\frac{i}{2 \pi}\right)^{N+1} d X_{0} \overline{d X}_{0} \ldots d X_{N} \overline{d X}_{N} .
\end{aligned}
$$

On se servira des relations de récurrence sur l'intégrale sur $\mathbb{C}^{N+1}$ et de (35) pour déduire des relations de récurrence pour l'intégrale sur $\mathbb{P}^{N}$. On définit

$$
I(Q, a, b)=\int_{\mathbb{C}^{N+1}} Q P^{a} \bar{P}^{b} e^{-S} d \mu
$$

pour $Q$ un polynôme homogène dans $\mathbb{C}\left[X_{0}, \ldots, X_{N}, \bar{X}_{0}, \ldots, \bar{X}_{N}\right]$ et $a, b$ des nombres complexes satisfaisant $\operatorname{Re}(a), \operatorname{Re}(b)>0, a-b \in \mathbb{Z}$.

Lemme 2.5. Pour tout polynôme homogène $Q$ de degré $q$ dans les variables $X_{0}, \ldots, X_{N}, \bar{X}_{0}, \ldots, \bar{X}_{N}$, on a

$$
\begin{gathered}
\left(\frac{a+b}{2} d+N+1+\frac{q}{2}\right) I(Q, a, b)=I(Q S, a, b) \\
(a+1)\left(\frac{a+b}{2} d+N+1+\frac{q+d-1}{2}\right) I\left(Q P_{X_{i}}, a, b\right) \\
+I\left(Q_{X_{i}} S, a+1, b\right)-\left(\frac{a+b}{2} d+N+1+\frac{q+d-1}{2}\right) I\left(Q \bar{X}_{i}, a+1, b\right)=0
\end{gathered}
$$


(39) $\quad(b+1)\left(\frac{a+b}{2} d+N+1+\frac{q+d-1}{2}\right) I\left(Q \bar{P}_{\bar{X}_{i}}, a, b\right)$

$+I\left(Q_{\bar{X}_{i}} S, a, b+1\right)-\left(\frac{a+b}{2} d+N+1+\frac{q+d-1}{2}\right) I\left(Q X_{i}, a, b+1\right)=0$.

Preuve. L'égalité

$$
\begin{aligned}
0= & \int_{\mathbb{C}^{N+1}} d\left(Q P^{a+1} \bar{P}^{b} e^{-S} \frac{d \mu}{d X_{i}}\right) \\
= & \int_{\mathbb{C}^{N+1}}(a+1) Q P_{X_{i}} P^{a} \bar{P}^{b} d \mu+\int_{\mathbb{C}^{N+1}} Q_{X_{i}} P^{a+1} \bar{P}^{b} e^{-S} d \mu \\
& +\int_{\mathbb{C}^{N+1}} Q P^{a+1} \bar{P}^{b}\left(-\bar{X}_{i}\right) d \mu
\end{aligned}
$$

s'écrit

(40)

$$
(a+1) I\left(Q P_{X_{i}}, a, b\right)+I\left(Q_{X_{i}}, a+1, b\right)-I\left(Q \bar{X}_{i}, a+1, b\right)=0 .
$$

En changeant $X_{i}$ avec $\bar{X}_{i}$ et $a$ avec $b$ on obtient

$$
(b+1) I\left(Q \bar{P}_{\bar{X}_{i}}, a, b\right)+I\left(Q_{\bar{X}_{i}}, a, b+1\right)-I\left(Q X_{i}, a, b+1\right)=0 .
$$

La relation (40) appliquée pour $a-1$ au lieu de $a$ et pour $Q X_{i}$ au lieu de $Q$ donne

$$
a I\left(Q X_{i} P_{X_{i}}, a-1, b\right)+I\left(X_{i} Q_{X_{i}}, a, b\right)-I\left(Q X_{i} \bar{X}_{i}, a, b\right)=0 .
$$

De même, (41) implique

$$
b I\left(Q \bar{X}_{i} \bar{P}_{\bar{X}_{i}}, a, b-1\right)+I\left(\bar{X}_{i} Q_{\bar{X}_{i}}, a, b\right)-I\left(Q X_{i} \bar{X}_{i}, a, b\right)=0 .
$$

La relation (37) résulte par l'addition des relations (42), (43) pour tous les $i=0, \ldots, M$, en tenant compte des relations

$$
\begin{array}{r}
\sum_{i=0}^{M} X_{i} Q_{X_{i}}+\sum_{i=0}^{M} \bar{X}_{i} Q_{\bar{X}_{i}}=q Q \\
\sum_{i=0}^{M} X_{i} P_{X_{i}}=d P \\
\sum_{i=0}^{M} \bar{X}_{i} \bar{P}_{\bar{X}_{i}}=d \bar{P}
\end{array}
$$

Par (37), on peut remplacer $I\left(Q_{X_{i}}, a+1, b\right)$ dans (40) par

$$
\left(\frac{a+b+1}{2} d+N+1+\frac{q-1}{2}\right)^{-1} I\left(Q_{X_{i}} S, a+1, b\right) .
$$

Cela prouve (38). De même, (37) et (41) impliquent (39). 
Soit $E$ le plus petit entier positif vérifiant

$$
E d+(d-2) \geq(N+1)(d-2)+1 .
$$

Tout polynôme homogène de bi-degré $(E d+(d-2), E d+(d-2))$ appartient, par le lemme 2.4, à l'idéal engendré par les dérivées partielles $\left(P_{t}\right)_{X_{i}}$ dans l'anneau $\mathbb{C}\left[X_{0}, \ldots, X_{N}, \bar{X}_{0}, \ldots, \bar{X}_{N}\right]$. On choisit une base $\mathcal{R}$ pour l'espace vectoriel de ces polynômes telle que chaque élément de la base est divisible par l'une des dérivées $\left(P_{t}\right)_{X_{i}}$.

De même, on choisit une base $\mathcal{T}$ pour l'espace vectoriel des polynômes bi-homogènes de degré $(E d-1, E d+d-1)$ telle que chaque élément de la base soit divisible par une des dérivées $\left(\bar{P}_{t}\right)_{\bar{X}_{i}}$. On choisit une base $\mathcal{Q}$ des polynômes bi-homogènes de degré $(E d, E d)$ qui contient le polynôme $Q_{1}=P_{t}^{E} \bar{P}_{t}^{E}$.

On note $I_{\mathcal{Q}}(s)$ le vecteur colonne formé par les intégrales $I(Q, s, s)$ pour tout $Q \in \mathcal{Q}, I_{\mathcal{R}}(s)$ le vecteur colonne formé par les intégrales $I(R, s, s)$ pour tout $R \in \mathcal{R}$ et $I_{\mathcal{T}}(s)$ le vecteur colonne formé par les intégrales $I(T, s+1, s)$ pour tout $T \in \mathcal{T}$.

Soit $Q \in \mathcal{Q}$. On applique la relation (37) pour $a=b=s$ successivement pour les polynômes $Q, Q S, \ldots, Q S^{d-3}$. On obtient

$(s d+N+1+E d) \ldots(s d+N+1+E d+d-3) I(Q, s, s)=I\left(Q S^{d-2}, s, s\right)$.

On exprime chacun des polynômes $Q S^{d-2}$ dans la base $\mathcal{R}$. On obtient

$$
I_{\mathcal{Q}}(s)=(s d+N+1+E d)^{-1} \ldots(s d+N+1+E d+d-3)^{-1} C I_{\mathcal{R}}(s)
$$

pour une matrice $C$ à coefficients constants en $s$.

Soit $R \in \mathcal{R}$. On applique (38) pour $a-b=s$ et pour une écriture de $R$, $R=P_{X_{i}} Q$. On exprime chacun des polynômes $Q_{X_{i}} S, Q \bar{X}_{i}$ dans la base $\mathcal{T}$. On obtient

$$
I_{\mathcal{R}}(s)=(s+1)^{-1}(s d+N+1+E d+d-2)^{-1} D(s) I_{\mathcal{T}}(s)
$$

pour une matrice $D(s)$ dont les coefficients sont polynômes de degré au plus 1 en $s$.

Par le même argument, en utilisant (39) pour $a=s+1, b=s$, on obtient

$$
I_{\mathcal{T}}(s)=(s+1)^{-1}(s d+N+1+E d+d-1) E(s) I_{\mathcal{Q}}(s+1)
$$

pour une matrice $E(s)$ dont les coefficients sont polynômes de degré au plus 1 en $s$.

$\mathrm{Au}$ total, les relations (45)-(47) donnent

$$
I_{\mathcal{Q}}(s)=\frac{1}{(s+1)^{2}} \cdot \frac{\Gamma(s d+E d+N+1)}{\Gamma(s d+E d+d+N+1)} B(s) I_{\mathcal{Q}}(s+1)
$$

pour une matrice $B(s)$ dont les coefficients sont polynômes en $s$ de degré au plus 2 . 
La dimension de l'espace $\mathcal{Q}$ est

$$
M=\left(\begin{array}{c}
E d+N \\
N
\end{array}\right)^{2} .
$$

La définition (44) de $E$ implique $E d+N \leq d+N(d-1)$, d'où l'estimation (33). On choisit une base $Q_{1}=P_{t}^{E} \bar{P}_{t}^{E}, \ldots, Q_{M}$ pour $\mathcal{Q}$ et on note

$$
I_{i}(s)=\int_{\mathbb{P}^{N}(\mathbb{C})} Q_{i}\left|P_{t}\right|^{2(s-E)} d \nu_{N}
$$

pour $i=1, \ldots, M$.

Les relations (48), (49), (35) impliquent

$$
\left(\begin{array}{c}
I_{1}(s) \\
\vdots \\
I_{M}(s)
\end{array}\right)=\frac{1}{(s-E+1)^{2}} B(s-E)\left(\begin{array}{c}
I_{1}(s+1) \\
\vdots \\
I_{M}(s+1)
\end{array}\right) .
$$

On note $A(s)=B(s-E)$. Évidemment $I_{1}(s)=K_{t}(s)$. Cela finit la preuve de la proposition 2.2 .

Preuve de la proposition 2.3. La relation (34) implique

$$
\left(\begin{array}{c}
I_{1}(s+i) \\
\vdots \\
I_{M}(s+i)
\end{array}\right)=\prod_{k=i}^{M-1}(s+k-E+1)^{-2} \cdot A^{i}(s)\left(\begin{array}{c}
I_{1}(s+M) \\
\vdots \\
I_{M}(s+M)
\end{array}\right)
$$

pour tout $i=0, \ldots, M$, où

$$
A^{i}(s)=\prod_{k=i}^{M-1} A(s+k) .
$$

On prend $A^{M}(s)=\mathrm{Id}$. La matrice $A^{i}(s)$ a les coefficients polynômes en $s$ de degré au plus $2(M-i)$, pour tout $i=0, \ldots, M$.

Il en résulte que

$$
\left(\begin{array}{c}
I_{1}(s) \\
\vdots \\
I_{1}(s+M)
\end{array}\right)=C(s)\left(\begin{array}{c}
I_{1}(s+M) \\
\vdots \\
I_{1}(s+M)
\end{array}\right)
$$

où $C_{i j}(s)=\prod_{k=i}^{M-1}(s+k-E+1)^{-2} \cdot A_{1 j}^{i}(s)$ pour tout $i=0, \ldots, M$ et tout $j=1, \ldots, M$. La fonction $A_{1 j}^{i}(s)$ est un polynôme en $s$ de degré au plus $2(M-i)$.

Soit $T$ le rang de la matrice $C(s)$. Si $T=0$, alors $I_{1}(s)=0$ et il n'y a rien à démontrer. Sinon, soit $\operatorname{det}\left(C_{I J}\right)$ un mineur non-nul, pour des sousensembles $I$ de $\{0, \ldots, M\}$ et $J$ de $\{1, \ldots, M\}$ de cardinal $T$. On ajoute un élément $i_{0}$ à $I$ et on note le nouveau ensemble $I_{0}$. Les fonctions $I_{1}(s+i)$ 
pour $i \in I_{0}$ vérifient

$$
\sum_{i \in I} b_{i}(s) I_{1}(s+i)=0,
$$

où

$$
b_{i}(s)= \pm \operatorname{det}\left(C_{I-\{i\}, J}\right)(s) .
$$

Le numérateur $a_{i}(s)$ de la fonction rationnelle $\pm \operatorname{det}\left(C_{I-\{i\}, J}\right)(s)$ est un polynôme en $s$ de degré au plus

$$
\sum_{j \in\{0, \ldots, \hat{i}, \ldots, M\}} 2(M-j)=M(M-1)+2 i .
$$

Le dénominateur de la fonction $b_{i}(s)$ est

$\prod_{j \in\{0, \ldots, \hat{i}, \ldots, M\}} \prod_{k=j}^{M-1}(s-E+k+1)^{2}=\prod_{k=1}^{i}(s-E+k)^{2 k} \cdot \prod_{k=i+1}^{M}(s-E+k)^{2 k-2}$.

3. Calculs explicites. Soit $N$ un entier positif et $d_{0}, \ldots, d_{N}$ des entiers positifs. On note $R_{\left(d_{0}, \ldots, d_{N}\right)}$ le résultant des $N+1$ polynômes en $N+1$ variables homogènes de degrés $d_{0}, \ldots, d_{N}$. C'est un polynôme multi-homogène sur l'espace

$$
\mathbb{P}^{\left(N ; d_{0}, \ldots, d_{N}\right)}=\mathbb{P}^{\left(\begin{array}{c}
d_{0}+N \\
N
\end{array}\right)} \times \ldots \times \mathbb{P}^{\left(\begin{array}{c}
d_{N}+N \\
N
\end{array}\right)}
$$

des coefficients de ces polynômes, qui s'annule aux points où les polynômes correspondant à ces coefficients ont un zéro commun dans $\mathbb{P}^{N}$. On s'intéresse au calcul de l'intégrale

$$
\zeta\left(R_{\left(d_{0}, \ldots, d_{N}\right)}, s\right)=\int_{\mathbb{P}^{\left(N ; d_{0}, \ldots, d_{N}\right)}(\mathbb{C})}\left|R_{\left(d_{0}, \ldots, d_{N}\right)}\right|^{2 s} d \nu_{\left(N ; d_{0}, \ldots, d_{N}\right)},
$$

où $d \nu_{\left(N ; d_{0}, \ldots, d_{N}\right)}$ est le produit des mesures invariantes et de volume 1 sur les facteurs.

La dérivée en $s=0$ de cette fonction a été calculée en [BGS], (4.3.39) :

$$
\frac{1}{2} \zeta^{\prime}\left(R_{\left(d_{0}, \ldots d_{N}\right)}, 0\right)=\left(\prod_{i=0}^{N} d_{i}\right)\left(\sigma_{N}-\frac{1}{2} \sum_{i=0}^{N} \frac{1}{d_{i}}\left(1+\frac{1}{2}+\ldots+\frac{1}{N_{i}}\right)\right) .
$$

On reprend ici deux calculs, dus à Maillot, de la fonction (55) dans le cas $N$ général, $d_{0}=\ldots=d_{N}=1$ et $N=1, d_{0}=1, d_{1}=d$ général.

3.1. La fonction zêta associée au résultant $R_{(1, \ldots, 1)}$

Lemme 3.1. Soit $k$ un entier positif, soient $d_{1}, \ldots, d_{k}$ et $N_{1}, \ldots, N_{k}$ des entiers positifs et $P$ un polynôme multi-homogène de degré $\left(d_{1}, \ldots, d_{k}\right)$ sur 
$\mathbb{P}^{N_{1}}(\mathbb{C}) \times \ldots \times \mathbb{P}^{N_{k}}(\mathbb{C})$. Alors

$$
\begin{aligned}
& \int_{\mathbb{P}^{N_{1}}(\mathbb{C}) \times \ldots \times \mathbb{P}^{N_{k}(\mathbb{C})}}|P|^{2 s} d \nu_{N_{1}} \ldots d \nu_{N_{k}} \\
&= \frac{\Gamma\left(N_{1}+1\right)}{\Gamma\left(s d_{1}+N_{1}+1\right)} \cdots \frac{\Gamma\left(N_{k}+1\right)}{\Gamma\left(s d_{k}+N_{k}+1\right)} \\
& \times \int_{\mathbb{C}^{N_{1}+1} \times \ldots \times \mathbb{C}^{N_{k}+1}}|P|^{2 s} e^{-S_{1}-\ldots-S_{k}} d \mu_{1} \ldots d \mu_{k},
\end{aligned}
$$

où $S_{1}, \ldots, S_{k}, d \mu_{1}, \ldots, d \mu_{k}$ sont définis par la formule (36).

Preuve. La relation résulte par l'application successive de (35) et par la formule de Fubini :

$$
\begin{aligned}
& \int_{\mathbb{P}^{N_{1}}(\mathbb{C}) \times \ldots \times \mathbb{P}^{N_{k}}(\mathbb{C})}|P|^{2 s} d \nu_{N_{1}} \ldots d \nu_{N_{k}} \\
= & \int_{\mathbb{P}^{N_{1}}(\mathbb{C}) \times \ldots \times \mathbb{P}^{N_{k-1}}(\mathbb{C}) \times \mathbb{C}^{N_{k}+1}}|P|^{2 s} d \nu_{N_{1}} \ldots d \nu_{N_{k-1}} d \mu_{k} \frac{\Gamma\left(N_{k}+1\right)}{\Gamma\left(s d_{k}+N_{k}+1\right)}=\ldots \\
= & \int_{\mathbb{P}^{N_{1}}(\mathbb{C}) \times \mathbb{C}^{N_{2}+1} \ldots \times \mathbb{C}^{N_{k}+1}}|P|^{2 s} d \nu_{N_{1}} d \mu_{2} \ldots d \mu_{k} \\
& \times \frac{\Gamma\left(N_{2}+1\right)}{\Gamma\left(s d_{2}+N_{2}+1\right)} \ldots \frac{\Gamma\left(N_{k}+1\right)}{\Gamma\left(s d_{k}+N_{k}+1\right)} \\
= & \int_{\mathbb{C}^{N_{1}+1} \times \ldots \times \mathbb{C}^{N_{k}+1}}|P|^{2 s} d \mu_{1} \ldots d \mu_{k} \cdot \frac{\Gamma\left(N_{1}+1\right) \ldots \Gamma\left(N_{k}+1\right)}{\Gamma\left(s d_{1}+N_{1}+1\right) \ldots \Gamma\left(s d_{k}+N_{k}+1\right)} .
\end{aligned}
$$

Lemme 3.2. Soit $f$ une fonction sur $\mathbb{C}^{N+1}$, invariante sous l'action $d u$ groupe $U(N+1)$ et vérifiant $f(\lambda v)=|\lambda|^{a} f(v)$, pour tout nombre complexe et tout vecteur $v \in \mathbb{C}^{N+1}$, pour un nombre complexe a. Alors

$$
\int_{\mathbb{C}^{N+1}} f e^{-S} d \mu=f(1,0, \ldots, 0) \frac{\Gamma(N+1+a s / 2)}{\Gamma(N+1)},
$$

où $S$ et $d \mu$ sont définis par la formule (36).

Preuve. On munit la sphère unité $S^{2 N+1} \subset \mathbb{C}^{N+1}$ de la métrique invariante sous l'action du groupe $U(N+1)$, normalisée, pour que l'aire de la sphère soit 1. La relation d'homogénéité $f(\lambda v)=|\lambda|^{a} f(v)$ implique

$$
\int_{\mathbb{C}^{N+1}} f e^{-s} d \mu=\frac{\Gamma(N+1+a s / 2)}{\Gamma(N+1)} \int_{S^{2 N+1}} f d S^{2 N+1}
$$

et la dernière intégrale donne $f(1,0, \ldots, 0)$ par l'invariance. 
La proposition suivante calcule l'intégrale (55) pour $d_{0}=\ldots=d_{N}=1$. Pour $i=0, \ldots, N$ on considère le polynôme linéaire $L_{i}=\sum_{j=0}^{N} a_{i j} X_{j}$ sur $\mathbb{P}^{N}$. On note $a_{i}$ le vecteur $\left(a_{i_{0}}, \ldots, a_{i_{N}}\right)$. Le résultant des polynômes $L_{i}$ sur $\mathbb{P}^{N}$ est

$$
R_{(1, \ldots, 1)}=\operatorname{det}_{i=0, \ldots, N}\left(a_{i}\right)=\operatorname{det}\left(a_{i j}\right)_{0 \leq i, j \leq N},
$$

un polynôme multi-homogène de multi-degré $(1, \ldots, 1)$ sur $\mathbb{P}^{N} \times \ldots \times \mathbb{P}^{N}$.

Proposition 3.3.

$$
\int_{\mathbb{P}^{N}(\mathbb{C}) \times \ldots \times \mathbb{P}^{N}(\mathbb{C})}|R(1, \ldots, 1)|^{2 s} d \nu_{N} \ldots d \nu_{N}=\prod_{k=1}^{N}\left(\frac{k}{s+k}\right)^{k} .
$$

Preuve. La relation (58) résulte de (59) et de la relation

$$
\int_{\mathbb{C}^{N+1} \times \ldots \times \mathbb{C}^{N+1}}\left|R_{(1, \ldots, 1)}\right|^{2 s} e^{-S_{0}-\ldots-S_{N}} d \mu_{0} \ldots d \mu_{N}=\prod_{k=1}^{N+1} \frac{\Gamma(s+k)}{\Gamma(k)}
$$

qu'on va prouver par induction. On a noté $d \mu_{i}$ la mesure $d \mu$ défini par (36) sur le $i$-ème facteur de $\mathbb{C}^{N+1} \times \ldots \times \mathbb{C}^{N+1}$ et $S_{i}=\sum_{j=0}^{N}\left|a_{i j}\right|^{2}$.

On définit la fonction $f: \mathbb{C}^{N+1} \rightarrow \mathbb{C}$ par la formule

$$
f\left(a_{0}\right)=\int_{\mathbb{C}^{N+1} \times \ldots \times \mathbb{C}^{N+1}}\left|\operatorname{det}\left(a_{0}, \ldots, a_{N}\right)\right|^{2 s} e^{-S_{1}-\ldots-S_{N}} d \mu_{1} \ldots d \mu_{N} .
$$

Elle satisfait aux conditions du Lemme 3.2 :

$$
\begin{aligned}
f\left(\lambda a_{0}\right)= & |\lambda|^{2 s} f\left(a_{0}\right), \\
f\left(\mu a_{0}\right)= & \int_{\mathbb{C}^{N+1} \times \ldots \times \mathbb{C}^{N+1}}\left|\operatorname{det}\left(u a_{0}, a_{1}, \ldots, a_{N}\right)\right|^{2 s} e^{-S_{1}-\ldots-S_{N}} d \mu_{1} \ldots d \mu_{N} \\
= & \int_{\mathbb{C}^{N+1} \times \ldots \times \mathbb{C}^{N+1}}|\operatorname{det} u|^{2 s}\left|\operatorname{det}\left(a_{0}, u^{-1} a_{1}, \ldots, u^{-1} a_{N}\right)\right|^{2 s} \\
& \times e^{-\left(u^{-1}\right)^{*} S_{1}-\ldots-\left(u^{-1}\right)^{*} S_{N}} \cdot\left(u^{-1}\right)^{*} d \mu_{1} \cdot \ldots \cdot\left(u^{-1}\right)^{*} d \mu_{N} \\
= & \int_{\mathbb{C}^{N+1} \times \ldots \times \mathbb{C}^{N+1}}\left|\operatorname{det}\left(a_{0}, \ldots, a_{N}\right)\right|^{2 s} e^{-S_{1}-\ldots-S_{N}} d \mu_{1} \ldots d \mu_{N}=f\left(a_{0}\right)
\end{aligned}
$$

pour tout $u \in U(N+1)$.

Il résulte du lemme 3.2 que

$$
\int_{\mathbb{C}^{N+1} \times \ldots \times \mathbb{C}^{N+1}}\left|\operatorname{det}\left(a_{0}, a_{1}, \ldots, a_{N}\right)\right|^{2 s} e^{-S_{0}-\ldots-S_{N}} d \mu_{0} \ldots d \mu_{N}
$$




$$
\begin{aligned}
& =\frac{\Gamma(s+N+1)}{\Gamma(N+1)} \int_{\mathbb{C}^{N+1} \times \ldots \times \mathbb{C}^{N+1}}\left|\operatorname{det}\left(e_{0}, a_{1}, \ldots, a_{N}\right)\right|^{2 s} e^{-S_{1}-\ldots-S_{N}} d \mu_{1} \ldots d \mu_{N} \\
& =\int_{\mathbb{C}^{N+1} \times \ldots \times \mathbb{C}^{N+1}}\left|\operatorname{det}\left(a_{i j}\right)_{1 \leq i, j \leq N}\right|^{2 s} e^{-S_{1}-\ldots-S_{N}} d \mu_{1} \ldots d \mu_{N} \frac{\Gamma(s+N+1)}{\Gamma(N+1)},
\end{aligned}
$$

où on a noté $e_{0}=(1,0, \ldots, 0)$.

Les termes $a_{1,0}, \ldots, a_{N, 0}$ n'apparaissent pas dans $\left|\operatorname{det}\left(a_{i j}\right)_{1 \leq i, j \leq N}\right|^{2 s}$.

Pour tout $i=1, \ldots, N$ on écrit $\mathbb{C}^{N+1}=\mathbb{C} \times \mathbb{C}^{N}$, le facteur $\mathbb{C}$ correspondant à la variable $a_{i, 0}$. On écrit $d \mu_{i}=d \mu_{i}^{0} d \mu_{i}^{1}$, où $d \mu_{i}^{0}, d \mu_{i}^{1}$ sont les mesures données par la formule (36) sur les facteurs de ce produit. On écrit $S_{i}=S_{i}^{0}+S_{i}^{1}$ où $S_{i}^{0}=\left|a_{i 0}\right|^{2}$ et $S_{i}^{1}=\sum_{j=1}^{N}\left|a_{i j}\right|^{2}$. On a

$$
\begin{aligned}
& \int_{\mathbb{C}^{N+1} \times \ldots \times \mathbb{C}^{N+1}}\left|\operatorname{det}\left(a_{i j}\right)_{1 \leq i, j \leq N}\right|^{2 s} e^{-S_{1}-\ldots-S_{N}} d \mu_{1} \ldots d \mu_{N} \\
& =\prod_{i=1}^{N}\left(\int_{\mathbb{C}} e^{-S_{i}^{0}} d \mu_{i}^{0}\right) \int_{\mathbb{C}^{N} \times \ldots \times \mathbb{C}^{N}}\left|\operatorname{det}\left(a_{i j}\right)\right|^{2 s} e^{-S_{1}^{1}-\ldots-S_{N}^{1}} d \mu_{1}^{1} d \mu_{N}^{1} .
\end{aligned}
$$

Par le lemme 3.2 appliqué à la fonction constante $f=1$, chacune des intégrales $\int_{\mathbb{C}} e^{-S_{i}^{0}} d \mu_{i}^{0}$ vaut 1 . Par l'hypothèse de récurrence, la dernière intégrale vaut $\prod_{k=1}^{N} \Gamma(s+k) / \Gamma(k)$. Donc les formules (60) et (61) prouvent (59).

3.2. La fonction zêta associée au résultant $R_{(1, n)}$. On considère maintenant $N=1$; on note $X$ et $Y$ les coordonnées sur $\mathbb{P}^{1}$. On considère les polynômes homogènes $a_{1} X+a_{0} Y$ et $b_{n} X^{n}+b_{n-1} X^{n-1} Y+\ldots+b_{0} Y^{n}$ sur $\mathbb{P}^{1}$. Leur résultant est $R_{(1, n)}=b_{0} a_{1}^{n}+b_{1} a_{0} a_{1}^{n-1}+\ldots+b_{n}\left(-a_{0}\right)^{n}$, polynôme bi-homogène de bi-degré $(n, 1)$ sur $\mathbb{P}^{1} \times \mathbb{P}^{n}$.

\section{Proposition 3.4.}

$$
\begin{aligned}
\int_{\mathbb{P}^{1}(\mathbb{C}) \times \mathbb{P}^{n}(\mathbb{C})}\left|R_{(1, n)}\right|^{2 s} d \nu_{1} d \nu_{n} \\
=\frac{n !}{(s+1) \ldots(s+n)} \int_{0}^{1}\left[t^{n}+t^{n-1}(1-t)+\ldots+(1-t)^{n}\right]^{s} d t .
\end{aligned}
$$

Preuve. Par le lemme 3.1,

$$
\begin{gathered}
\int_{\mathbb{P}^{1}(\mathbb{C}) \times \mathbb{P}^{n}(\mathbb{C})}\left|R_{(1, n)}\right|^{2 s} d \nu_{1} d \nu_{n}=\frac{\Gamma(2)}{\Gamma(n s+2)} \cdot \frac{\Gamma(n+1)}{\Gamma(s+n+1)} \\
\times \int_{\mathbb{C}^{2} \times \mathbb{C}^{n+1}}\left|b_{0} a_{1}^{n}-b_{1} a_{0} a_{1}^{n-1}+\ldots+b_{n}\left(-a_{0}\right)^{n}\right|^{2 s} e^{-\left|a_{0}\right|^{2}-\left|a_{1}\right|^{2}-\sum_{i=0}^{n}\left|b_{i}\right|^{2}} d \mu .
\end{gathered}
$$


On intègre d'abord sur les fibres $\mathbb{C}^{n+1}$, pour tout $\left(a_{0}, a_{1}\right) \in \mathbb{C}^{2}$. L'expression $b_{0} a_{1}^{n}-b_{1} a_{0} a_{1}^{n-1}+\ldots+b_{n}\left(-a_{0}\right)^{n}$ est linéaire en $b_{0}, \ldots, b_{n}$, donc par le théorème 1.3.1 de [Ca-M],

$$
\begin{aligned}
\int_{\mathbb{C}^{n+1}}\left|b_{0} a_{1}^{n}-b_{1} a_{0} a_{1}^{n-1}+\ldots+b_{n}\left(-a_{0}\right)^{n}\right|^{2 s} e^{-\sum_{i=0}^{n}\left|b_{i}\right|^{2}} d \mu \\
=\left(\left|a_{1}\right|^{2 n}+\left|a_{1}\right|^{2 n-2}\left|a_{0}\right|^{2}+\ldots+\left|a_{0}\right|^{2 n}\right)^{s} \Gamma(s+1) .
\end{aligned}
$$

On est réduit au calcul de l'intégrale

$$
\int_{\mathbb{C}^{2}}\left(\left|a_{1}\right|^{2 n}+\left|a_{1}\right|^{2 n-2}\left|a_{0}\right|^{2}+\ldots+\left|a_{0}\right|^{2 n}\right)^{s} e^{-\left|a_{0}\right|^{2}-\left|a_{1}\right|^{2}} d \mu .
$$

Cette intégrale ne dépend que des modules $\left|a_{0}\right|,\left|a_{1}\right|$. Elle est donc égale à

$$
4 \int_{0}^{\infty} \int_{0}^{\infty}\left(X^{2 n}+X^{2 n-2} Y^{2}+\ldots+Y^{2 n-2}\right) e^{-X^{2}-Y^{2}} X Y d X d Y .
$$

En coordonnées polaires $X=r \cos \theta, Y=r \sin \theta$, l'intégrale (65) s'écrit

$$
\begin{gathered}
4 \int_{0}^{\infty} \int_{0}^{\pi / 2} d r d \theta e^{-r^{2}} r^{2 s}\left(\cos ^{2 n} \theta+\cos ^{2 n-2} \theta \sin \theta+\ldots+\sin ^{2 n} \theta\right)^{s} r^{3} \sin \theta \cos \theta \\
\quad=4 \int_{0}^{\infty} e^{-r^{2}} r^{2 n s+3} d r \int_{0}^{\pi / 2}\left(\cos ^{2 n} \theta+\ldots+\sin ^{2 n} \theta\right)^{s} \sin \theta \cos \theta d \theta \\
=\Gamma(n s+2) \int_{0}^{1}\left(t^{n}+t^{n-1}(1-t)+\ldots+(1-t)^{n}\right)^{s} d t
\end{gathered}
$$

(on a fait le changement des variables $t=\sin ^{2} \theta$ dans la dernière intégrale).

Les relations (63)-(66) impliquent

$$
\begin{array}{rl}
\int_{\mathbb{P}^{1}(\mathbb{C}) \times \mathbb{P}^{n}(\mathbb{C})}\left|R_{(1, n)}\right|^{2 s} & d \nu_{1} d \nu_{n} \\
& =\frac{\Gamma(n+1) \Gamma(s+1)}{\Gamma(s+n+1)} \int_{0}^{1}\left[t^{n}+\ldots+(1-t)^{n}\right]^{s} d t .
\end{array}
$$

DÉFINITION 3.5 ([La], [A]). Soit $n$ un entier positif, soient $a, c, b_{1}, \ldots, b_{n}$ des nombres complexes et $x_{1}, \ldots, x_{n}$ des nombres complexes de module plus petit que 1. La fonction de Lauricella $F_{D}$ de paramètres $a, c, b_{1}, \ldots, b_{n}$ et de variables $x_{1}, \ldots, x_{n}$ est la somme de la série absolument convergente

$$
\begin{aligned}
F_{D}\left(a ; b_{1}, \ldots b_{n} ; c \mid\right. & \left.x_{1}, \ldots, x_{n}\right) \\
= & \sum_{m_{1}, \ldots, m_{n} \geq 0} \frac{(a)_{m_{1}+\ldots+m_{n}}\left(b_{1}\right)_{m_{1}} \ldots\left(b_{n}\right)_{m_{n}}}{(c)_{m_{1}+\ldots+m_{n}} m_{1} ! \ldots m_{n} !} x_{1}^{m_{1}} \ldots x_{n}^{m_{n}} .
\end{aligned}
$$


On a utilisé la notation $(a)_{m}=a(a+1) \ldots(a+m-1)$ pour un nombre complexe $a$ et pour un entier $m \geq 0$. Par convention $(a)_{0}=1$.

Proposition 3.6.

$$
\int_{\mathbb{P}^{1}(\mathbb{C}) \times \mathbb{P}^{n}(\mathbb{C})}\left|R_{(1, n)}\right|^{2 s} d \nu_{1} d \nu_{n}
$$

$=\frac{n !}{(s+1)_{n}} F_{D}\left(1 ;-s, \ldots,-s ; \frac{3}{2} \mid \frac{1}{2}+\frac{1}{2} \cos \frac{2 \pi}{n+1}, \ldots, \frac{1}{2}+\frac{1}{2} \cos \frac{2[n / 2] \pi}{n+1}\right)$.

Preuve. Montrons d'abord que

$$
t^{n}+t^{n-1}(1-t)+\ldots+(1-t)^{n}=\prod_{k=1}^{[n / 2]}\left[\left(t^{2}-t\right)\left(2+2 \cos \frac{2 k \pi}{n+1}\right)+1\right] \text {. }
$$

Les deux expressions sont des polynômes de degré $2[n / 2]$ en $t$, dont le coefficient constant est égal à 1 . Montrons qu'ils ont les mêmes racines.

Une racine pour le polynôme $t^{n}+\ldots+(1-t)^{n}$ est une racine pour le polynôme $t^{n+1}-(1-t)^{n+1}$, différente de la racine $t=1 / 2$. Une telle racine s'écrit $\zeta /(1+\zeta)$, où $\zeta=\exp \frac{2 i k \pi}{n+1}$ pour un $k=1, \ldots, n$. On calcule

$$
\left(t-\frac{\zeta}{1+\zeta}\right)\left(t-\frac{\bar{\zeta}}{1+\bar{\zeta}}\right)=t^{2}-t+\frac{1}{2+2 \cos \frac{2 k \pi}{n+1}} .
$$

Par le changement des variables $v=4(t-1 / 2)^{2}$ on obtient

$$
\begin{aligned}
& \int_{0}^{1}\left[t^{n}+t^{n-1}(1-t)+\ldots+(1-t)^{n}\right]^{s} d t \\
& \quad=\frac{1}{2} \int_{0}^{1} \prod_{k=1}^{n / 2]}\left[1-\left(\frac{1}{2}+\frac{1}{2} \cos \frac{2 k \pi}{n+1}\right)(1-v)\right]^{s} v^{-1 / 2} d v .
\end{aligned}
$$

Pour chaque $k=1, \ldots,[n / 2]$,

$$
\begin{aligned}
{\left[1-\left(\frac{1}{2}+\frac{1}{2} \cos \right.\right.} & \left.\left.\frac{2 k \pi}{n+1}\right)(1-v)\right]^{s} \\
& =\sum_{m_{k} \geq 0} \frac{(-s)_{m_{k}}}{m_{k} !}\left(\frac{1}{2}+\frac{1}{2} \cos \frac{2 k \pi}{n+1}\right)^{m_{k}}(1-v)^{m_{k}}
\end{aligned}
$$

et

$$
\begin{aligned}
\int_{0}^{1}(1-v)^{m_{1}+\ldots+m_{k}} & v^{-1 / 2} d v=\frac{\Gamma\left(m_{1}+\ldots+m_{k}+1\right) \Gamma(1 / 2)}{\Gamma\left(m_{1}+\ldots+m_{k}+3 / 2\right)} \\
= & \frac{\Gamma(1 / 2) \cdot(1)_{m_{1}+\ldots+m_{k}}}{\Gamma(3 / 2) \cdot(3 / 2)_{m_{1}+\ldots+m_{k}}}=2 \frac{(1)_{m_{1}+\ldots+m_{k}}}{(3 / 2)_{m_{1}+\ldots+m_{k}}} .
\end{aligned}
$$

Les formules (61), (68)-(70) impliquent l'égalité de l'énoncé. 
3.3. La fonction zêta associée au résultant $R_{(1,2)}$. La relation $(56)$ et le calcul de la proposition 3.3 pourraient suggérer que $\zeta(P, s)$ est une combinaison linéaire de produits de fonctions gamma si $P$ est un polynôme résultant $R_{\left(d_{0}, \ldots d_{N}\right)}$. La proposition suivante montre que cela n'est pas vrai dans le cas $N=1, d_{0}=1, d_{1}=2$.

Proposition 3.7. La fonction $s \rightarrow \int_{\mathbb{P}^{1}(\mathbb{C}) \times \mathbb{P}^{2}(\mathbb{C})}\left|R_{(1,2)}\right|^{2 s} d \nu_{1} d \nu_{2}$ n'est pas une combinaison linéaire de fonctions de type

$$
\prod_{i=1}^{M} \Gamma\left(\alpha_{i} s+\beta_{i}\right)^{\gamma_{i}} a_{i}^{s}
$$

où $\alpha_{i}, \beta_{i} \in \mathbb{C}, \gamma_{i} \in \mathbb{Z}, a_{i} \in \mathbb{R}_{+}$.

Preuve. Par la relation (61) il suffit de prouver que la fonction

$$
I(s)=\int_{0}^{1}\left[t^{2}+t(1-t)+(1-t)^{2}\right]^{s} d t
$$

n'est pas une combinaison linéaire de fonctions de type (71). La fonction $I(s)$ satisfait l'équation

$$
1=(2 s+1) I(s)-\frac{3}{2} s I(s-1)
$$

car

$$
I(s)=\int_{0}^{1}\left(1-t+t^{2}\right)^{s} d t=\int_{0}^{1}\left[\left(t-\frac{1}{2}\right)^{2}+\frac{3}{4}\right]^{s} d t=2 \int_{0}^{1 / 2}\left(u^{2}+\frac{3}{4}\right)^{s} d u
$$

et

$$
\begin{aligned}
\frac{1}{2}= & {\left[u\left(u^{2}+\frac{3}{4}\right)^{s}\right]_{0}^{1 / 2}=\int_{0}^{1 / 2}\left[u\left(u^{2}+\frac{3}{4}\right)^{s}\right]^{\prime} d u } \\
= & \int_{0}^{1 / 2}\left[\left(u^{2}+\frac{3}{4}\right)^{s} \cdot 1+s\left(2 u^{2}+\frac{3}{2}\right)\left(u^{2}+\frac{3}{4}\right)^{s-1}\right. \\
& \left.-s \frac{3}{2}\left(u^{2}+\frac{3}{4}\right)^{s-1}\right] d u \\
= & \frac{2 s+1}{2} I(s)-\frac{3}{4} s I(s-1)
\end{aligned}
$$

On soustrait l'égalité (72) d'elle même pour $s, s+1$. On obtient

$$
(3 s+3) I(s)-(7 s+12) I(s+1)+(4 s+10) I(s+2)=0 .
$$

On regarde l'équation (74) pour $s \in \mathbb{N}$. On utilise l'algorithme de $[\mathrm{Pe}]$ pour voir si cette équation admet des solutions de la forme (71). On introduit les définitions suivantes. 
DÉfinition 3.8. (a) Une série $s \rightarrow I(s)$ s'appelle hypergéométrique si pour $s \in \mathbb{N}$ assez grand $I(s) \neq 0$ et $I(s+1) / I(s)$ est une fraction rationnelle en $s$.

(b) Une combinaison linéaire finie de séries hypergéométriques s'appelle forme fermée.

(c) Deux séries $I(s), J(s)$ s'appellent rationnellement équivalentes si $J(s) / I(s)$ est une fraction rationnelle pour $s \in \mathbb{N}$ assez grand.

La proposition 5.2 et le théorème 5.1 de $[\mathrm{Pe}]$ affirment que toute forme fermée $a$ s'écrit d'une manière unique comme

$$
a=a_{1}+\ldots+a_{k}
$$

pour des séries hypergéométriques non rationnellement équivalentes $a_{1}, \ldots$ $\ldots, a_{k}$, et que la forme $a$ satisfait à l'équation aux différences homogène $L a=0$ si et seulement si $L a_{i}=0$ pour tout $i=1, \ldots, k$.

Il suffit donc de chercher les solutions hypergéométriques pour (74).

Pour une telle solution $I(s)$, on définit $S(s)=I(s+1) / I(s)$. L'équation (74) implique

$$
(4 s+10) S(s+1) S(s)-(7 s+12) S(s)+3 s+3=0,
$$

équation dont on cherche les solutions fonctions rationnelles en $s$.

Par le lemme 3.1 de $[\mathrm{Pe}]$, toute fonction rationnelle $S(s)$ s'écrit d'une manière unique dans la forme

$$
S(s)=Z \frac{A(s)}{B(s)} \cdot \frac{C(s+1)}{C(s)},
$$

pour une constante $Z$ et des polynômes $A(s), B(s), C(s)$ vérifiant :

- $A(s), B(s)$ sont des polynômes môniques, $k \in \mathbb{N}$,

- les polynômes $A(s), B(s+k)$ n'ont pas de racines communes pour tout

- les polynômes $A(s), C(s)$ n'ont pas de racines communes,

- les polynômes $B(s), C(s+1)$ n'ont pas de racines communes.

Avec cette écriture l'équation (75) s'écrit

$$
\begin{aligned}
Z^{2}(4 s+10) \frac{A(s+1)}{B(s+1)} C(s+2) & \\
& +Z(-7 s-12) C(s+1)+(3 s+3) \frac{B(s)}{A(s)} C(s)=0 .
\end{aligned}
$$

Cette équation implique que le polynôme $A(s)$ divise $3 s+3$, d'où les possibilités $A(s)=1, A(s)=s+1$, et que le polynôme $B(s+1)$ divise $4 s+10$, d'où les possibilités $B(s)=1, B(s)=s+3 / 2$.

Dans chacun de ces quatre cas, on cherche une solution polynômiale $C(s)$ pour (76). L'étude des coefficients dominants des polynômes $C(s+1)$, 
$C(s+2), C(s)$ donne d'abord une équation polynômiale pour la constante $Z$ et après une équation linéaire pour le degré du polynôme $C(s)$.

En faisant l'analyse de tous ces cas, on obtient une seule solution, donnée par $A(s)=s+1, B(s)=s+3 / 2, Z=3 / 4, C$ un polynôme constant. Cela donne

$$
I(s)=\alpha\left(\frac{3}{4}\right)^{s} \frac{\Gamma(s+1)}{\Gamma(s+3 / 2)}
$$

pour $\alpha$ une constante complexe. Mais cette solution pour l'équation homogène (74) n'est pas une solution pour l'équation initiale (72).

3.4. La fonction zêta d'un polynôme quadratique. Dans cette sous-section on reprend les calculs de [Da] sur l'intégrale $\zeta(P, s)$ quand $P$ est une polynôme quadratique et on exprime $\zeta(P, s)$ comme somme de "séries hypergéométriques généralisées".

A cause de l'invariance unitaire, on peut se réduire au cas où $P=$ $\sum_{i=0}^{N} a_{i} X_{i}^{2}$. On note $A_{i}=a_{i} \bar{a}_{i}$ pour $i=0, \ldots, N$. On suppose $N$ impair et $0<A_{0}<A_{1}<\ldots<A_{N}$. La relation (18) et le théorème 2.1 de [Da] impliquent

$$
\zeta(P, s)=\frac{\Gamma(N+1)}{\Gamma(2 s+N+1)} \Gamma(s+1)^{2} \cdot 4^{s} \frac{1}{2 i \pi} \int_{\gamma_{1}} \frac{t^{s-1+(N+1) / 2} d t}{\prod_{i=0}^{N}\left(t-A_{i}\right)^{1 / 2}},
$$

où $\gamma_{1}$ est un chemin injectif fermé dont l'intérieur contient les points $A_{i}$, mais pas 0 . On rappelle la notation $(a)_{m}=a(a+1) \ldots(a+m-1)$ pour tout entier positif $m,(a)_{0}=1$. On note $c(m)=(1 / 2)_{m}$ pour tout nombre naturel $m$.

Proposition 3.9. On a

$$
\begin{aligned}
\frac{1}{2 i \pi} \int_{\gamma_{1}} \frac{t^{s-1+(N+1) / 2} d t}{\prod_{i=0}^{N}\left(t-A_{i}\right)^{1 / 2}} \\
\quad=I_{N-1, N}-I_{N-3, N-2}+I_{N-5, N-4}-\ldots+(-1)^{(N+1) / 2} I_{0,1}
\end{aligned}
$$

avec

$$
\begin{aligned}
I_{2 k, 2 k+1}= & \frac{A_{2 k+1}^{s-1+(N+1) / 2}}{\prod_{i=0}^{2 k-1}\left(A_{2 k+1}-A_{i}\right)^{1 / 2} \cdot \prod_{i=2 k+2}^{N}\left(A_{i}-A_{2 k}\right)^{1 / 2}} \\
& \times \sum_{m_{0}, \ldots, m_{2 \hat{k}+1}, \ldots, m_{N} \geq 0} \frac{\prod_{i=0}^{N} c\left(m_{i}\right) \cdot(-s+1-(N+1) / 2)_{m_{2 k}}}{c(2 k) c(2 k+1) m_{0} ! \ldots m_{2 k} ! m_{2 k+2} ! \ldots m_{N} !} \\
& \times \frac{c\left(\sum_{i=0}^{2 k} m_{i}\right) c\left(\sum_{i=2 k+2}^{N} m_{i}\right)}{\left(\sum_{i=0}^{2 k} m_{i}+\sum_{i=2 k+2}^{N} m_{i}\right) !}
\end{aligned}
$$




$$
\begin{aligned}
& \times \prod_{i=0}^{2 k-1}\left(\frac{A_{2 k+1}-A_{2 k}}{A_{2 k+1}-A_{i}}\right)^{m_{i}} \cdot\left(\frac{A_{2 k+1}-A_{2 k}}{A_{2 k+1}}\right)^{m_{2 k}} \\
& \times \prod_{i=2 k+2}^{N}\left(\frac{A_{2 k+1}-A_{2 k}}{A_{i}-A_{2 k}}\right)^{m_{i}} .
\end{aligned}
$$

Pre u ve. L'intégrale sur $\gamma_{1}$ est indépendante du chemin $\gamma_{1}$ qui entoure les points $A_{i}$. S'il tend vers l'intervalle $\left[A_{0}, A_{N}\right]$, en tenant compte de la détermination choisie pour les expressions $t^{s-1+(N+1) / 2}$ et $\prod_{i=0}^{N}\left(t-A_{i}\right)^{1 / 2}$, l'intégrale tend vers

$$
(-1)^{(N+1) / 2} I_{01}+(-1)^{(N-1) / 2} I_{23}+\ldots+I_{N-1, N},
$$

où

$$
I_{2 k, 2 k+1}=\frac{1}{\pi} \int_{A_{2 k}}^{A_{2 k+1}} \frac{t^{s-1+(N+1) / 2} d t}{\prod_{i=0}^{2 k}\left(t-A_{i}\right)^{1 / 2} \cdot \prod_{i=2 k+1}^{N}\left(A_{i}-t\right)^{1 / 2}} .
$$

Par le changement de variable

$$
t=A_{2 k}+u\left(A_{2 k+1}-A_{2 k}\right)=A_{2 k+1}-(1-u)\left(A_{2 k+1}-A_{2 k}\right)
$$

on obtient

$$
\begin{aligned}
I_{2 k, 2 k+1}= & \frac{1}{\pi} \int_{0}^{1} \frac{(1-u)^{-1 / 2} u^{-1 / 2}\left[A_{2 k+1}-(1-u)\left(A_{2 k+1}-A_{2 k}\right)\right]^{s-1+(N+1) / 2}}{\prod_{i=0}^{2 k-1}\left(A_{2 k+1}-A_{i}-(1-u)\left(A_{2 k+1}-A_{2 k}\right)\right)^{1 / 2}} \\
& \times\left(\prod_{i=2 k+2}^{N}\left(A_{i}-A_{2 k}-u\left(A_{2 k+1}-A_{2 k}\right)\right)\right)^{-1} d u \\
= & \frac{1}{\pi} \frac{A_{2 k+1}^{s-1+(N+1) / 2}}{\prod_{i=0}^{2 k-1}\left(A_{2 k+1}-A_{i}\right)^{1 / 2} \cdot \prod_{i=2 k+2}^{N}\left(A_{i}-A_{k}\right)^{1 / 2}} \\
& \times \int_{0}^{1}\left[1-(1-u) \frac{A_{2 k+1}-A_{2 k}}{A_{2 k+1}}\right]^{s-1+(N+1) / 2} \\
& \times \prod_{i=0}^{2 k-1}\left[1-(1-u) \frac{A_{2 k+1}-A_{2 k}}{A_{2 k+1}-A_{i}}\right]^{-1 / 2} \\
& \times \prod_{i=2 k+2}^{N}\left[1-u \frac{A_{2 k+1}-A_{2 k}}{A_{i}-A_{2 k}}\right]^{-1 / 2} \cdot u^{-1 / 2}(1-u)^{-1 / 2} d u .
\end{aligned}
$$

Par un calcul analogue aux relations (69), (70), on trouve le résultat annoncé. On utilise le fait que $\Gamma(1 / 2) \Gamma(1 / 2)=\pi$.

Remerciements. Je remercie C. Soulé pour m'avoir guidé pendant la réalisation de ce travail, V. Maillot pour m'avoir communiqué les résultats des propositions 3.3 et 3.4, A. Duval pour m'avoir indiqué le changement 
des variables (68) et l'article [Pe], et D. Barsky, J.-P. Bézivin, F. Loeser, C. Sabbah, G. Turinici et D. Zagier pour les conversations très instructives.

\section{References}

[A] P. Appell, Fonctions hypergéométriques et hypersphériques, Gauthier-Villars, 1926.

[Be-Y] C. A. Berenstein and A. Yger, Green currents and analytic continuation, prépublication, 1995.

[Be] I. N. Bernshteŭn, Modules over a ring of differential operators. Study of the fundamental solutions of equations with constant coefficients, Funktsional. Anal. i Prilozhen. 5 (1971), no. 2, 1-16 (in Russian).

[BGS] J.-B. Bost, H. Gillet and C. Soulé, Heights of projective varieties and positive Green forms, J. Amer. Math. Soc. 7 (1994), 903-1027.

[Ca-M] J. Cassaigne et V. Maillot, Hauteurs des hypersurfaces et fonctions zêta d'Igusa, preprint ENS, 1994-1995.

[Da] N. Dan, La hauteur des quadratiques, prépublication, 1997.

[GKZ] I. M. Gelfand, M. M. Kapranov and A. V. Zelevinsky, Generalized Euler integrals and A-hypergeometric functions, Adv. in Math. 84 (1990), 255-271.

[GZK] I. M. Gelfand, A. V. Zelevinsky and M. M. Kapranov, Hypergeometric functions and toral manifolds, Funktsional. Anal. i Prilozhen. 23 (1989), no. 2, 12-26 (in Russian).

[GH] P. Griffiths and J. Harris, Principles of Algebraic Geometry, Wiley-Interscience, 1978.

[La] G. Lauricella, Sulle funzioni ipergeometriche a più variabili, Rend. Circ. Mat. Palermo 7 (1893), 111-158.

[Pe] M. Petkovšek, Hypergeometric solutions of linear recurrences with polynomial coefficients, J. Symbolic Comp. 14 (1992), 243-264.

[Y] A. Yger, Résidus, courants résiduels et courants de Green, dans : Géométrie complexe (Paris, 1992), Actualités Sci. Ind. 1438, Hermann, Paris, 1996, 123-147.

Institut Galilée

Université Paris XIII

Av. J.-B. Clement

93430 Villetaneuse, France
Institut de Mathématiques de l'Académie Roumaine P.O. Box 1-764

70700 Bucureşti, Romania E-mail: ndan@linux.imar.ro 